\title{
A Patient with ARDS and Cardiogenic Shock Caused by Streptococcus Pyogenes Successfully Treated by V-A-V ECMO
}

\author{
Thang Nguyen Quang ${ }^{1 *}$, Tuan Nguyen Dang², Binh Le Van², Quang Nguyen Ngoc'2, Bao Le Thai', and Giang Truong Tran² \\ ${ }^{1}$ Head, Department of Intensive Care Unit and Emergency, Vinmec International Hospital, Vietnam \\ ${ }^{2}$ Department of Intensive Care Unit, Vinmec International Hospital, Vietnam
}

*Corresponding author: Thang Nguyen Quang, Head, Department of Intensive Care Unit and Emergency, Vinmec International Hospital, Vietnam, E-mail: v.thangnq@vinmec.com

Received: 27 Jun, 2019 | Accepted: 15 Jul, 2019 | Published: 22 Jul, 2019

Citation: Quang TN, Dang TN, Van BL, Ngoc QN, Thai BL, et al. (2019) A Patient with ARDS and Cardiogenic Shock Caused by Streptococcus Pyogenes Successfully Treated by V-A-V ECMO. J Surg Open Access 5(3): dx.doi.org/10.16966/2470-0991.184

Copyright: (C) 2019 Quang TN, et al. This is an open-access article distributed under the terms of the Creative Commons Attribution License, which permits unrestricted use, distribution, and reproduction in any medium, provided the original author and source are credited.

\begin{abstract}
We performed VV ECMO for a 68-year-old man who had ARDS caused by Streptococcus pyogenes. After 2 days of treatment he had acute cardiogenic shock with refractory VT. Echocardiography showed EF 30\%. We planned transition from VV ECMO to VAV ECMO to support cardiopulmonary function. An additional return cannula $(15 \mathrm{Fr})$ was inserted from the left femoral Artery, which was connected to the circuit branch from the original returning cannula. Then we titrated the arterial and venous inflow by applying an adjustable clamp on the venous inflow limb to keep the optimal femoral artery flow, he was successfully switched from VV to VAV ECMO showed on ABG and hemodynamic stable. After 10 days of V-A-V treatment, he was recovered, stopped ECMO supporting and discharged hospital after 47 days.
\end{abstract}

Keywords: Extracorporeal membrane oxygenation; Venoarterial; Venovenous; Myocarditis; Streptococcus pyogenes; ARDS

\section{Introduction}

With the development of Extracorporeal Membrane Oxygenation (ECMO) technique recently, the patients with Acute Respiratory Distress Syndrome (ARDS), cardiogenic shock which failed with traditional therapies treatment was recovered with ECMO therapy. Almost patients were indicated with V-V ECMO mode to provide respiratory support and V-A ECMO mode to provide both respiratory and hemodynamic support. In patient supported with V-A ECMO mode sometimes cause differential hypoxia (lower $\mathrm{PaO}_{2}$ in the upper body and higher $\mathrm{PaO}_{2}$ in the lower body) because of the development of cardiac function after ECMO but simultaneous has severe impairment of pulmonary function (Figure 1) [1].

It is difficult to treat the patient with both Lung and Heart impairment with only $\mathrm{V}-\mathrm{V}$ or $\mathrm{V}-\mathrm{A}$ ECMO mode because those therapies can't provide the same good enough oxygen level to whole body. Recently, some case reports described about the Veno-ArterioVenous (V-A-V) ECMO mode which reported in patients with lung and heart failure [1] and had successful treatment. Although this technique can solve problems which only $\mathrm{V}-\mathrm{V}$ mode or $\mathrm{V}-\mathrm{A}$ mode can't do on specific patient but it is complicated procedure and only indicated for cases with combined heart and lung failure, such as severe left ventricular failure with secondary pneumonia or right heart decompensation during ARDS [2].

Therefore, in the beginning we indicated V-V ECMO for our Patients with ARDS first, then he had heart failure (right and left ventricular failure) developing and became hemodynamic unstable, we chose the V-A-V ECMO mode by adding an arterial cannula to provide circulatory support including maintaining systemic blood pressure and unloading the right ventricular [2].

\section{Case Report}

68 -year-old man presented at ED with fever $\left(>40^{\circ} \mathrm{C}\right)$, hypoxic condition with arterial oxygen tension $\left(\mathrm{PaO}_{2}\right)$ of $58 \mathrm{mmHg}, \mathrm{SPO}_{2}$ $90 \%$ on $15 \mathrm{~L} / \mathrm{min}$ of oxygen via mask with a reservoir. Chest X-ray: diffuse bilateral ground glass opacities (Figures 2 and 3), diagnosed with severe Pneumonia received empirical antibiotics (Meropenem 3 $\mathrm{g} /$ day and Levofloxacin $750 \mathrm{mg}$ /day, admitted to Intensive Care Unit (ICU) for Noninvasive ventilation. On the second day he developed hypoxemia and endotracheal intubation required but he continued refractory to mechanical ventilation and became ARDS with arterial blood gas analysis showed P/F: 78 despite providing maximum positive end expiratory pressure of $12 \mathrm{cmH}_{2} \mathrm{O}$ on the third day (Figure 2). V-V mode ECMO was introduced immediately to recover severe hypoxic condition. At the first day of ECMO, he was subsequently initiated with circuit flow of $4.0 \mathrm{~L} / \mathrm{min}$ and sweep gas of $3.0 \mathrm{~L} / \mathrm{min}$ of oxygen. $\mathrm{FiO}_{2}$ of 100 , lung protective ventilation provided, tracheal endoscopy performed (Figure 4) and the sputum cultures found that had Streptococcus pyogenes and smoothed with current antibiotic (levofloxacin). We gave more Clindamycin for him on the fourth day of treatment. After ECMO applying, his condition was stable, chest $\mathrm{X}$-ray and arterial blood gas analysis showed quickly improvement, his infection condition had been controlled. 


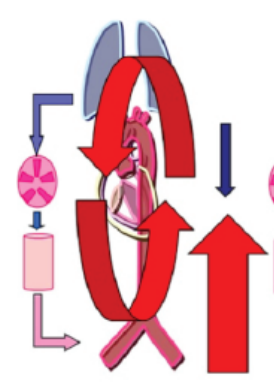

(A)

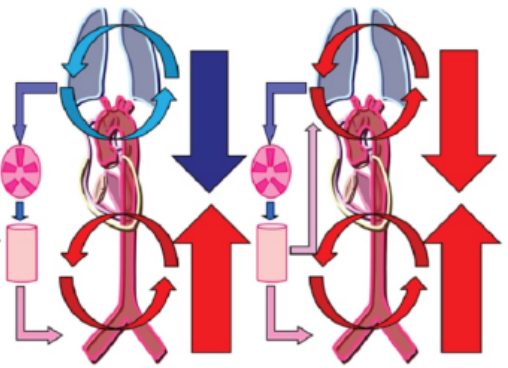

(C)
Figure 1: Schematic diagrams of circulation during Extracorporeal Membrane Oxygenation (ECMO). The diagrams indicate circulation in the case of typical veno-arterial ECMO (A), differential hypoxia (B), and veno-arterial venous ECMO (C). Red or pink arrows=oxygenated blood; blue arrows=deoxygenated blood; downward arrows=cardiac output; upward arrows=retrograde ECMO output.

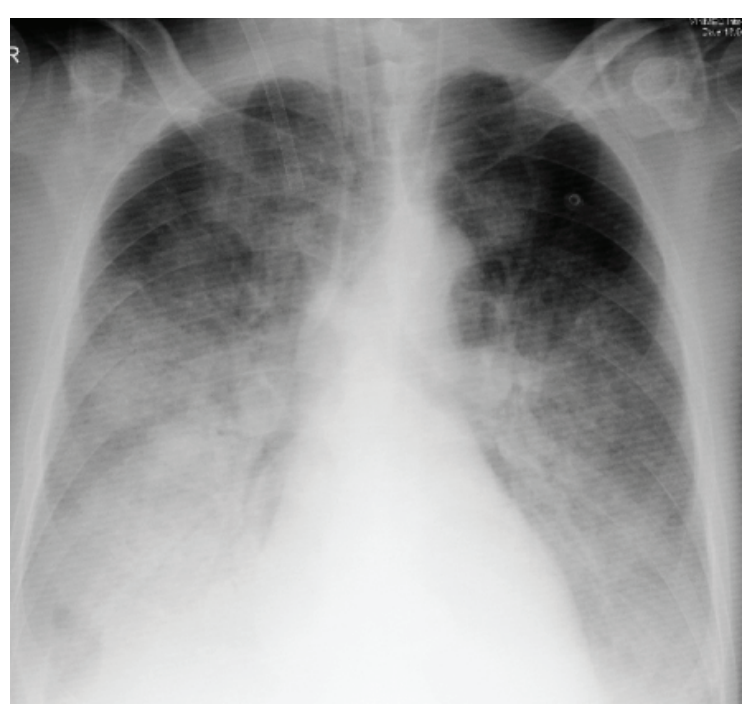

Figure 2: Chest X-Ray in ECMO indicated day.

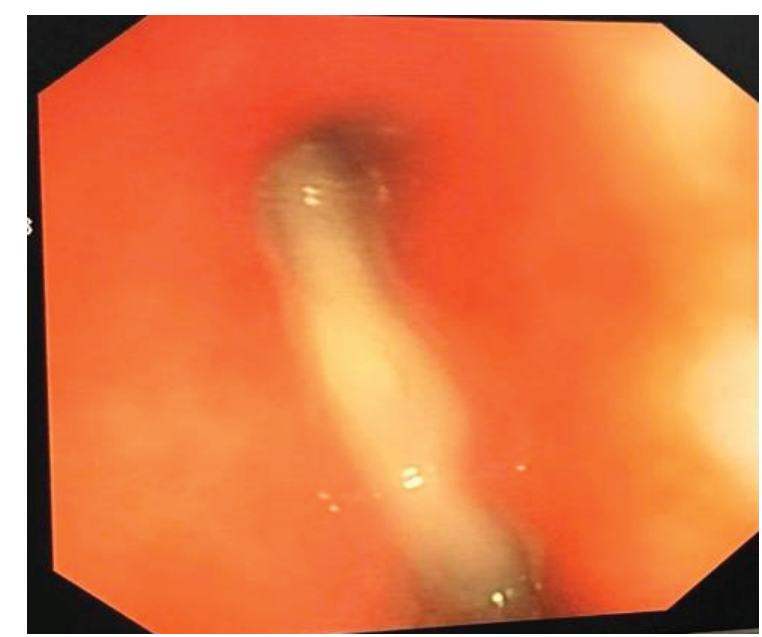

Figure 3: Tracheal Endoscopy, sputum in all tracheal branches.

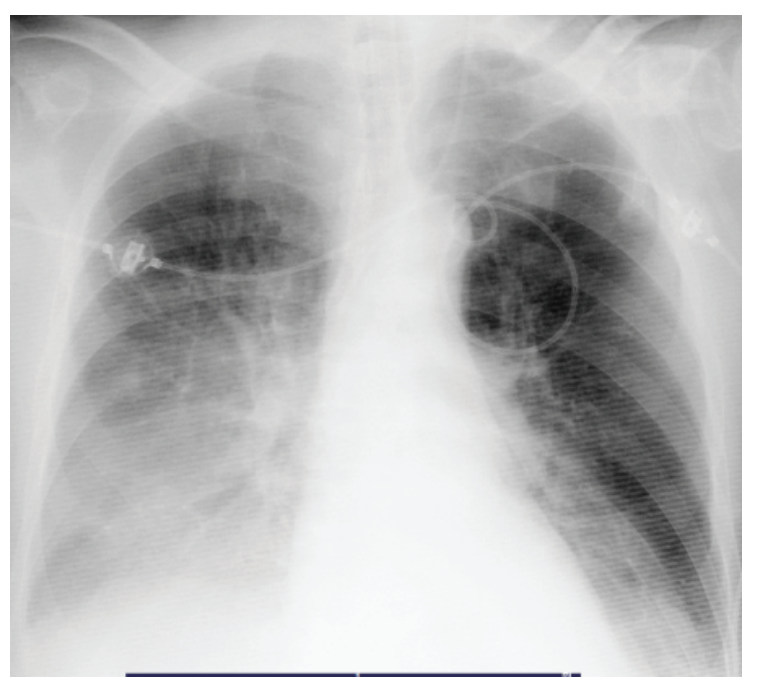

Figure 4: Chest X-Ray in ECMO Stopped day.

From third day of V-V ECMO, the patient developed acute worsening hypoxia and had refractory ventricular tachycardia couldn't be treated with electric shock and antiarrhythmic drugs, cardiac echocardiography showed severe left ventricular systolic dysfunction, severe ventricular hypokinesis with an ejection fraction of $30 \%$. For maintaining systemic blood pressure and unloading the heart, we decided to change V-V ECMO Mode to V-A-V ECMO Mode which supported lung and unloaded heart (Figure 5). Following V-A-V established, it was important to provide an individual demand of arterialized blood flow for each supplying cannulas, which will also vary during therapy [3]. So we had to adjust the balance by "Adjustable clamp" and "flow sensor measurement" with purpose provided an optimal artery blood gas and blood pressure for him (Figures 5 and 6).

After changing from $\mathrm{V}-\mathrm{V}$ to $\mathrm{V}-\mathrm{A}-\mathrm{V}$ ECMO support, gradually, chest $\mathrm{X}$-ray and arterial blood gas analysis showed improvement (Figures 4,7-9), and could be decreased Dobutamin, Norepinephrin dosage. We slowly changed the flow between venous outflow and artery outflow depending on blood pressure and artery blood gas (Figure 8), cardiac function monitored to prevent right ventricular overload during adjusting flow. Right hand $\mathrm{SPO}_{2}$ continuously kept upper 96\% which purposed of avoiding "harlequin syndrome" [4]. After 10 days (VAV1-10) of V-A-V ECMO mode, the cardiac function improved, we completely changed to V-V ECMO mode before stopped ECMO support 2 days later. After 47 days of treatment the patient was discharged from hospital.

\section{Discussion}

Extracorporeal Membrane Oxygenation (ECMO) can be a life saving therapy in patients with refractory severe respiratory failure or cardiac failure. Severe Acute Respiratory Distress Syndrome (ARDS) still has a high-mortality rate, but ECMO may be able to improve the outcome. Use of ECMO for respiratory failure has been increasing since 2009. Initiation of ECMO for adult ARDS should be considered when conventional therapy cannot maintain adequate oxygenation [5].

Streptococcus pyogenes is life-threatening infections include scarlet fever, bacteremia, Pneumonia, necrotizing fasciitis, cardiac dysfunction or Streptococcal Toxic Shock Syndrome (StrepTSS). 


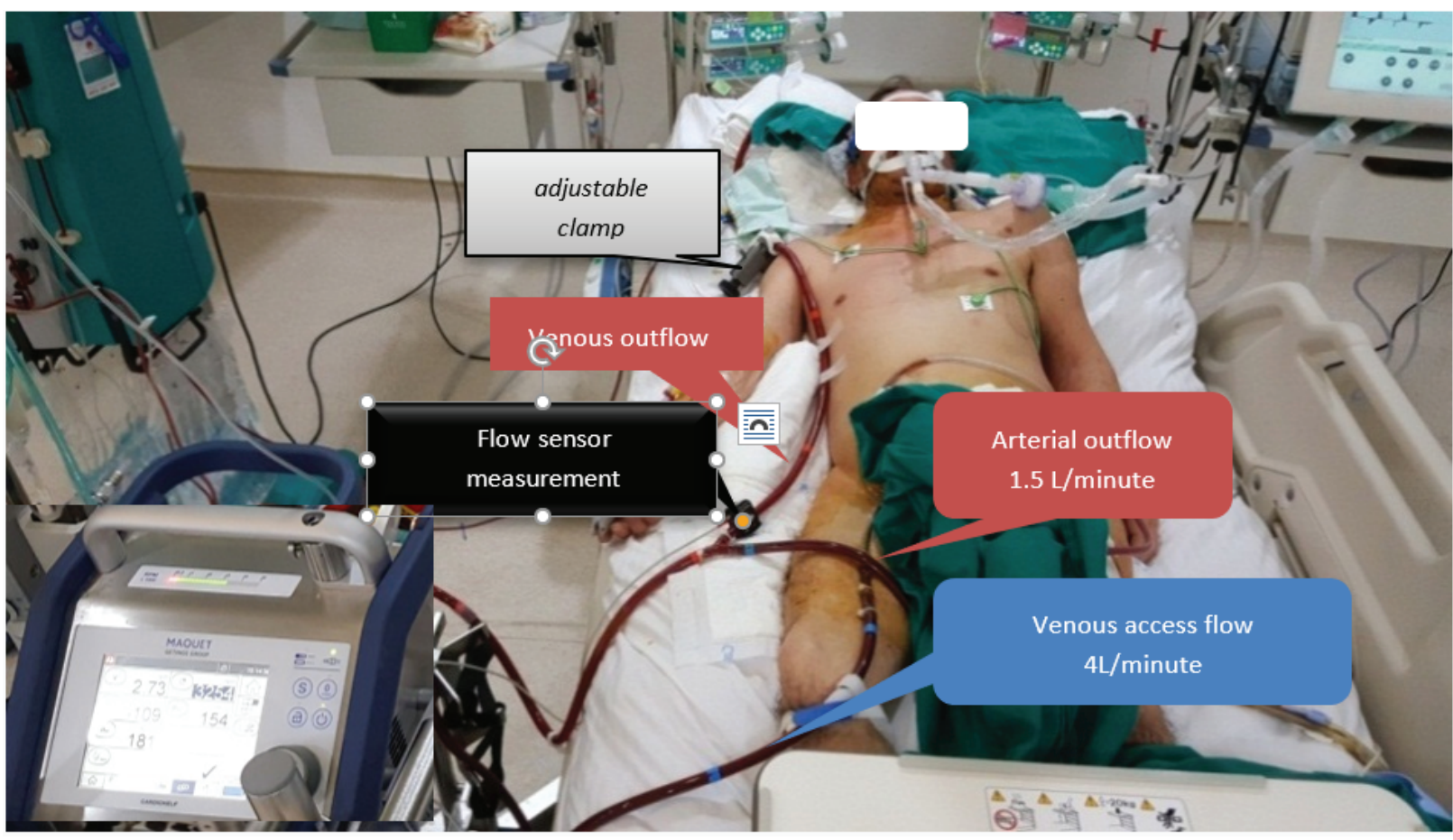

Figure 5: Setup V-A-V ECMO on patient.

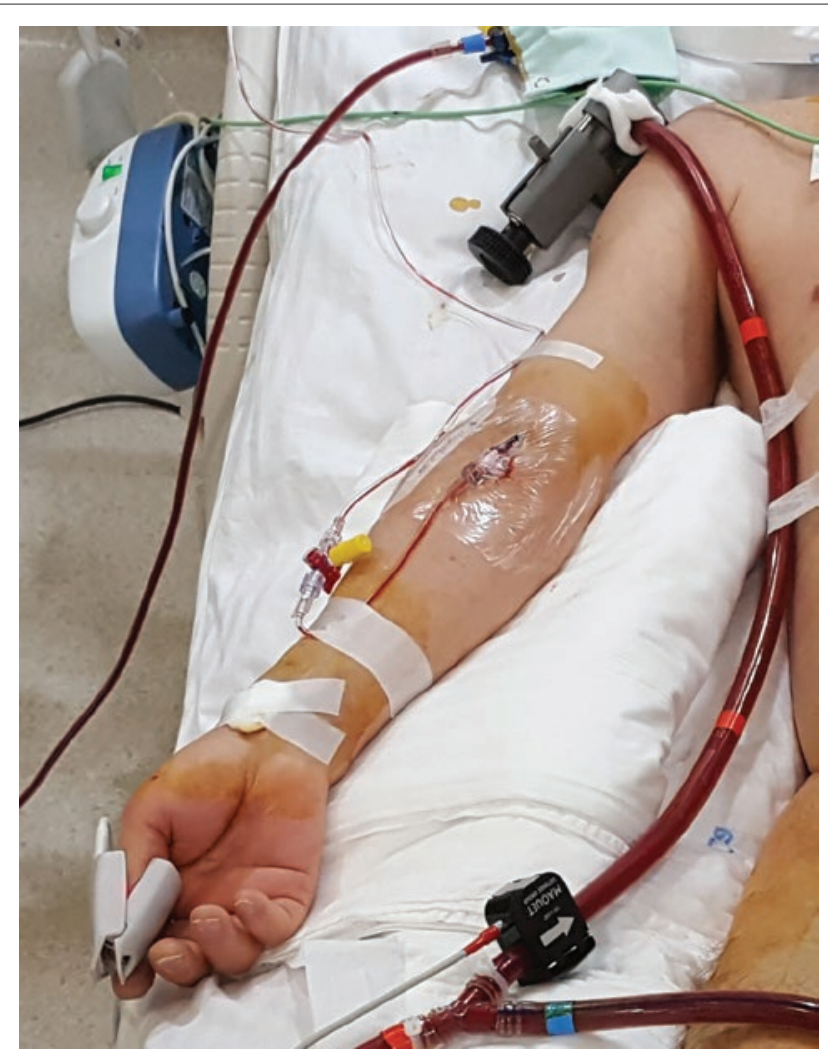

Figure 6: Flow sensor measurement and adjustable clamp at Vinmec International Hospital.
$\mathrm{V}-\mathrm{A}-\mathrm{V}$ ECMO is effective in treating patients with simultaneous acute respiratory failure and cardiogenic shock.

Our Patients was diagnosed with ARDS which could not be cured with high technique ventilator support we initially indicated V-V ECMO mode for him, after 3 days, he had refractory heart failure especially on right heart lead to unstable hemodynamic condition later, the V-V ECMO was switched to V-A-V ECMO in which, an arterial cannula was added on right femoral artery to provide circulatory support simultaneous decrease the right heart loading, maintain systemic blood pressure. In this our ECMO case for the cannulas strategy, we had to divide the arterial outflow to two ways which one part towards the aorta (Atery Outflow) through right femoral artery that support the hemodynamic and the second part towards the right atrium (Venous Outflow) providing the high oxygen level blood mixing with poor oxygen blood from natural flow, both components will be pumped through to lung (Figure 10), Both flows are controlled by sensor measurement and adjustable clamp device (Figure 6). With clamp adjustment device, we can turn to circulatory support by decreasing the venous flow or turn to respiratory support by decreasing the artery flow following the patient conditions demand during ECMO therapy [6], or we can provide both respiratory and circulatory support at the same time.

Routine control of right and left ventricular filling and systolic function by trans thoracic echocardiography, continuously monitoring $\mathrm{ScvO}_{2}$, lactate levels and saturation levels in the right radial artery during V-A-V ECMO are important to balance the venous return and arterial return to provide an optimal flow for the patient. Furthermore, we believe that VAV ECMO could be the treatment of choice for differential hypoxia resulting from VA ECMO $[7,8]$. 


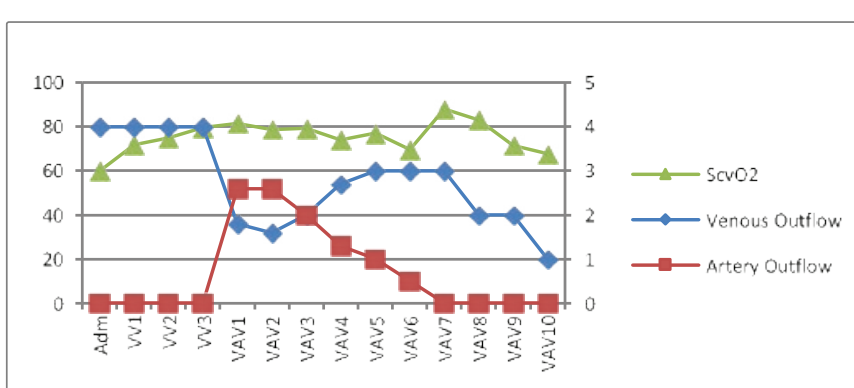

Figure 7: The changing in $\mathrm{ScvO}_{2}$ between venous outflow and artery outflow during treatment.
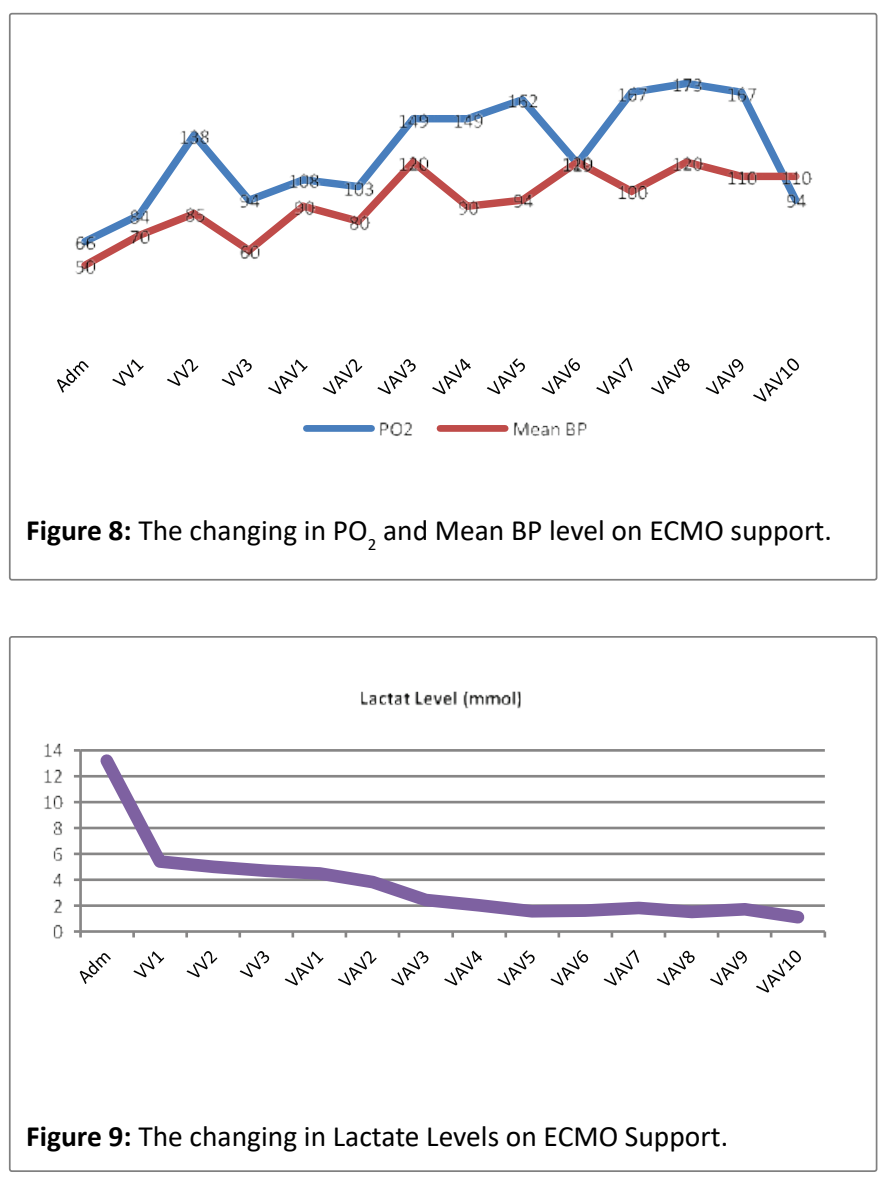

\section{Conclusion}

To our knowledge, this is the first successful clinical report of management of ARDS with Cardiogenic shock using V-A-V ECMO in Vietnam. We suggest that veno-arterial-venous ECMO could be choice for severe cardiopulmonary dysfunction treatment. It is important to switch flow balance between venous outflow and artery outflow, how to get unloading heart, $\mathrm{ScvO}_{2}$ and good saturation levels in the right radial artery.

\section{References}

1. Kitamura M, Shibuya M, Kurihara H, Akimoto T, Endo M, et al. (1997) Effective cross-circulation technique of venoarterial bypass for differential hypoxia condition. Artif Organs 21: 786788.

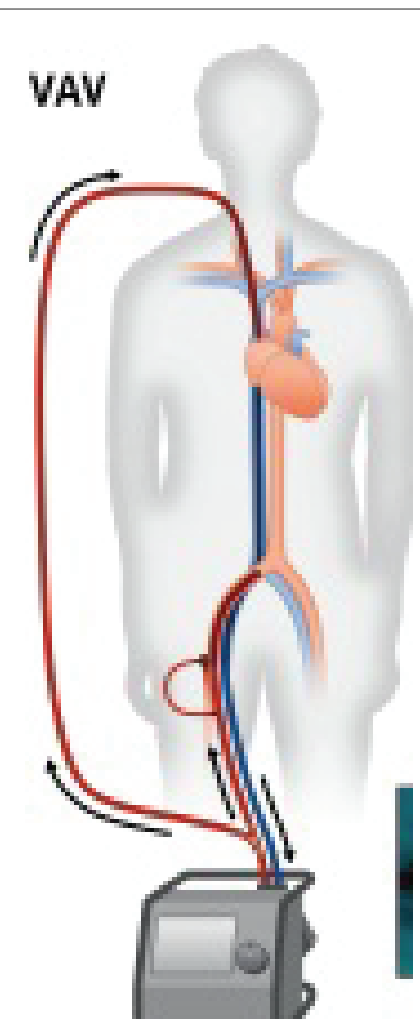

Figure 10: Veno-arterio-venous ECMO (VAV). Flows through the supplying cannulas are balanced by using an adjustable clamp (inset, black arrow) and a separate flow sensor (inset, Gray arrow).

2. Hou X, Yang X, Du Z, Xing J, Li H, et al. (2015) Superior vena cava drainage improves upper body oxygenation during veno-arterial extracorporeal membrane oxygenation in sheep. Crit Care 19: 68.

3. Napp LC, Kühn C, Hoeper MM, Vogel-Claussen J, Haverich A, et al. (2016) Cannulation strategies for percutaneous extracorporeal membrane oxygenation in adults. Clin Res Cardiol 105: 283-296.

4. Rupprecht L, Lunz D, Philipp A, Lubnow M, Schmid C (2015) Pitfalls in percutaneous ECMO cannulation. Heart Lung Vessel 7: $320-326$

5. Aokage T, Palmér K, Ichiba S, Takeda S (2015) Extracorporeal membrane oxygenation for acute respiratory distress syndrome. Journal of Intensive Care 3: 17.

6. Javidfar J, Brodie D, Costa J, Miller J, Jurrado J, et al. (2012) Subclavian artery cannulation for venoarterial extracorporeal membrane oxygenation. ASAIO J 58: 494-498.

7. Choi JH, Kim SW, Kim YU, Song-Yi K, Ki-Seok K, et al. (2014) Application of veno-arterial-venous extracorporeal membrane oxygenation in differential hypoxia. Multidiscip Respir Med 9: 55.

8. Australia and New Zealand Extracorporeal Membrane Oxygenation (ANZ ECMO) Influenza Investigators, Davies A, Jones D, Bailey M, Beca J, et al. (2009) Extracorporeal Membrane Oxygenation for 2009 Influenza A(H1N1) Acute Respiratory Distress Syndrome. JAMA 302: 1888-1895. 